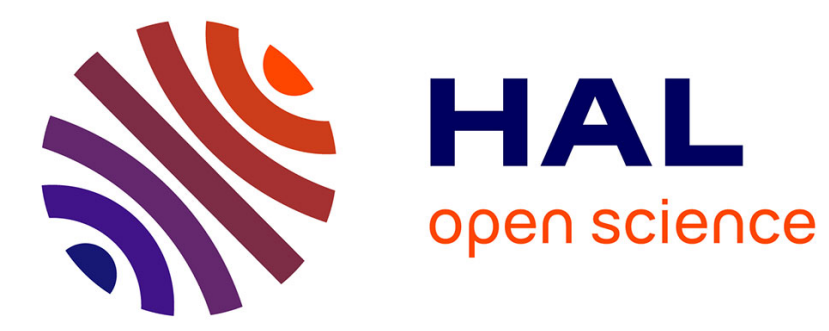

\title{
Mass flow rate and permeability measurements in microporous media
}

\author{
Martin Victor Johansson, Fabrice Testa, Imen Zaier, Pierre Perrier, Jean \\ Philippe Bonnet, Philippe Moulin, Irina Graur
}

\section{To cite this version:}

Martin Victor Johansson, Fabrice Testa, Imen Zaier, Pierre Perrier, Jean Philippe Bonnet, et al.. Mass flow rate and permeability measurements in microporous media. Vacuum, 2018, 158, pp.75-85. 10.1016/j.vacuum.2018.09.030 . hal-01888007

\section{HAL Id: hal-01888007 https://hal.science/hal-01888007}

Submitted on 8 Mar 2019

HAL is a multi-disciplinary open access archive for the deposit and dissemination of scientific research documents, whether they are published or not. The documents may come from teaching and research institutions in France or abroad, or from public or private research centers.
L'archive ouverte pluridisciplinaire HAL, est destinée au dépôt et à la diffusion de documents scientifiques de niveau recherche, publiés ou non, émanant des établissements d'enseignement et de recherche français ou étrangers, des laboratoires publics ou privés. 


\section{$1 \quad$ Mass flow rate and permeability measurements in}

microporous media

${ }_{3}$ M.V. Johansson ${ }^{1}$, F. Testa ${ }^{1}$, I. Zaier ${ }^{1}$, P. Perrier ${ }^{1}$, J.P. Bonnet ${ }^{2}$, P. Moulin ${ }^{2}$,

4

5

6

7

8

${ }^{1}$ Aix-Marseille Université, CNRS, IUSTI UMR 7343, 5 rue E. Fermi, 13453,

Marseille,France

Membranaires (EPM), Europôle de l'Arbois, BP80, Pavillon Laennec, Hall C, 13545 Aix en

Provence Cedex, France

\section{Abstract}

The transient method of the mass flow rate measurements through a microporous media is developed and analyzed. This method is based on the constant volume technique and the exponential fit of the pressure evolution in each tank which allows calculating the permeability directly. The pressure relaxation time, a single fitting parameter, is introduced and its behaviors are analyzed in a large pressure range. By measuring the pressure relaxation time for one gas, the permeability of a microporous sample can be derived for the other gases. With the actual experimental setup, we measured the mass flow rate through the microporous media in the range $5 \cdot 10^{-7}-5 \cdot 10^{-12}\left[\mathrm{~kg} \mathrm{~s}^{-1}\right]$ and the permeability in the range $10^{-14}-10^{-11}\left[\mathrm{~m}^{2}\right]$. 


\section{Introduction}

The determination of the permeability of porous media like the micro and nanoporous membranes or ultra-tight shale-gas reservoirs is still a challenge up to now. The low porous membranes find a broad application in medicine [1] and biotechnology for separation and filtration [2]. The recent development of low porous ceramic media with high thermal, chemical and structural stability and the ability to have catalytic properties has opened up new horizons for this kind of membrane applications, for example, in high-temperature gas separation and catalytic reactions [3]. Another type of porous media, the ultra-tight shale-gas reservoirs of tiny pores (in nanoscale) play a significant role in securing hydrocarbon energy because of their potential to offset declines in conventional gas production [4]. In all of these type of applications, the porous media permeability has to be known.

Permeability is a measure of how readily a fluid can flow through a porous material [5]. Gas permeability is an important parameter to understand the transport characteristics of a fluid through a porous medium, which can be obtained from the mass or volume flow rate. For the determination of low permeability, either the steady-state or the transient methods can be used. The steady-state method needs a precise flow meter to measure very slow flow, so when the permeability is very low the conventional gas flowmeters may be inappropriate [6]. Therefore, the transient "pulse-decay" or "draw-down" techniques, [7], [8], [9], [1], [6], are also used to determine the low permeabilities. By using these techniques, the permeability can be calculated directly from the pressure variations in time, without going first through the mass flow rate measurements [8], [1].

The primary objective of the present work is to develop the transient method to measure the pressure evolution in time in high and low-pressure tanks generated by the gas flow through a homogeneous porous medium. This experimental methodology, based on the constant volume technique, was initially developed for the isothermal and non-isothermal measurements of the mass flow rate through the microchannels [1], [1], [1]. We provide here the physical justifications of the exponential fitting of the pressure variations with time as well as the physical conditions of its implementation. From the measured pressure variations in time, the mass flow rate through the porous medium is deduced. It is shown that the gas permeability can be easily obtained directly from the pressure variations with time without going first through the mass flow rate calculations. The main advantages of the proposed approach are: its simplicity, the possibility of further extraction of the Klinkenberg coefficient and average pore size, and, finally, its further generalization for the case of temperature gradient driven flows.

The paper is organized as follows. After a brief introduction, the experimental apparatus and methodology are presented in Section 2. Then, in Section 3, the relation between the pressure variations in the tanks and a porous medium permeability is established by introducing the pressure relaxation time, which properties are analyzed in detail in Section 4. The behaviors of the measured 
mass flow rate and permeability are discussed in next two Sections. The paper is closed with final comments and conclusions.

\section{Experimental methodology}

\subsection{Experimental apparatus}

The experimental setup is a high vacuum system capable of measuring up to 5 decades of pressure. In the presented experiment the mean pressure is varied from $75 \mathrm{~Pa}$ up to $131 \mathrm{kPa}$. This large pressure measurement range is achieved by using three pairings of four capacitance diaphragm manometers (CDM) with full-scales: $133 \mathrm{kPa}-133 \mathrm{kPa}, 133 \mathrm{kPa}-13.3 \mathrm{kPa}$ and $13.3 \mathrm{kPa}-1.33 \mathrm{kPa}$. Four high purity gas bottles with test gases, Helium, Neon, Nitrogen, Argon (Air Liquide, France) are used. The pumping is performed by a two-stage diaphragm vacuum pump (DVP) and a turbomolecular pump (TMP), see the schematic of the experimental setup in Fig. 1. Each side of the porous medium is connected to two reservoirs of volumes $V_{1}$ and $V_{2}$ for the high and low-pressure, respectively. Both tanks' volumes, including the volumes of the valves, connecting tubes and pressure sensors, are measured accurately, and these volumes are equal to $V_{1}=255.8 \pm 5.5 \mathrm{~cm}^{3}$ and $V_{2}=238.8 \pm 5.1 \mathrm{~cm}^{3}$, for the high and low-pressure tanks, respectively. Several leakage tests were performed for this setup showing the absence of the detectable increase in pressure, measured with the lowest F.S. pressure sensor of $1.33 \mathrm{kPa}$ over a period of 30 minutes. In addition, for all realized measurements we did not detected any linear increase of the mean pressure.

Two microporous samples, used in the experiments and mentioned in the following as the first and second discs, have a cylindrical shape (disc) with the same diameter and thickness (in main flow direction) equal to $D=9.5 \pm 0.01 \mathrm{~mm}$ and $L=2.3 \pm 0.01 \mathrm{~mm}$, respectively. The structure of these microporous discs is the same as that used to support the active layers of micro-to-ultra filtration ceramic membranes.

For such ceramic microporous medium, depending on manufacturer, the porosity is in the range $15-30 \%$ with pore diameter ranging from 1 to 10 $\mu \mathrm{m}$. The total volume of each porous disc is $0.14 \mathrm{~cm}^{3}$, so by taking $30 \%$ of porosity a gas volume inside the medium is approximately $0.047 \mathrm{~cm}^{3}$, which is much smaller than the volume of each tank.

The experiments are performed within a narrow temperature range, excluding any heat source in an environment. The temperature is measured using the thermocouple with the accuracy of $0.6 \mathrm{~K}$.

\subsection{Mass flow rate measurements}

The constant volume technique, used previously for the measurements of the mass flow rate through the microchannels [1], [1], [1], [1], was implemented here to measure the mass flow rate through different samples of a microporous ceramic medium. This technique allows deducing the mass flow rate from the pressure variation in time. The mass flow rate through a microporous medium is 


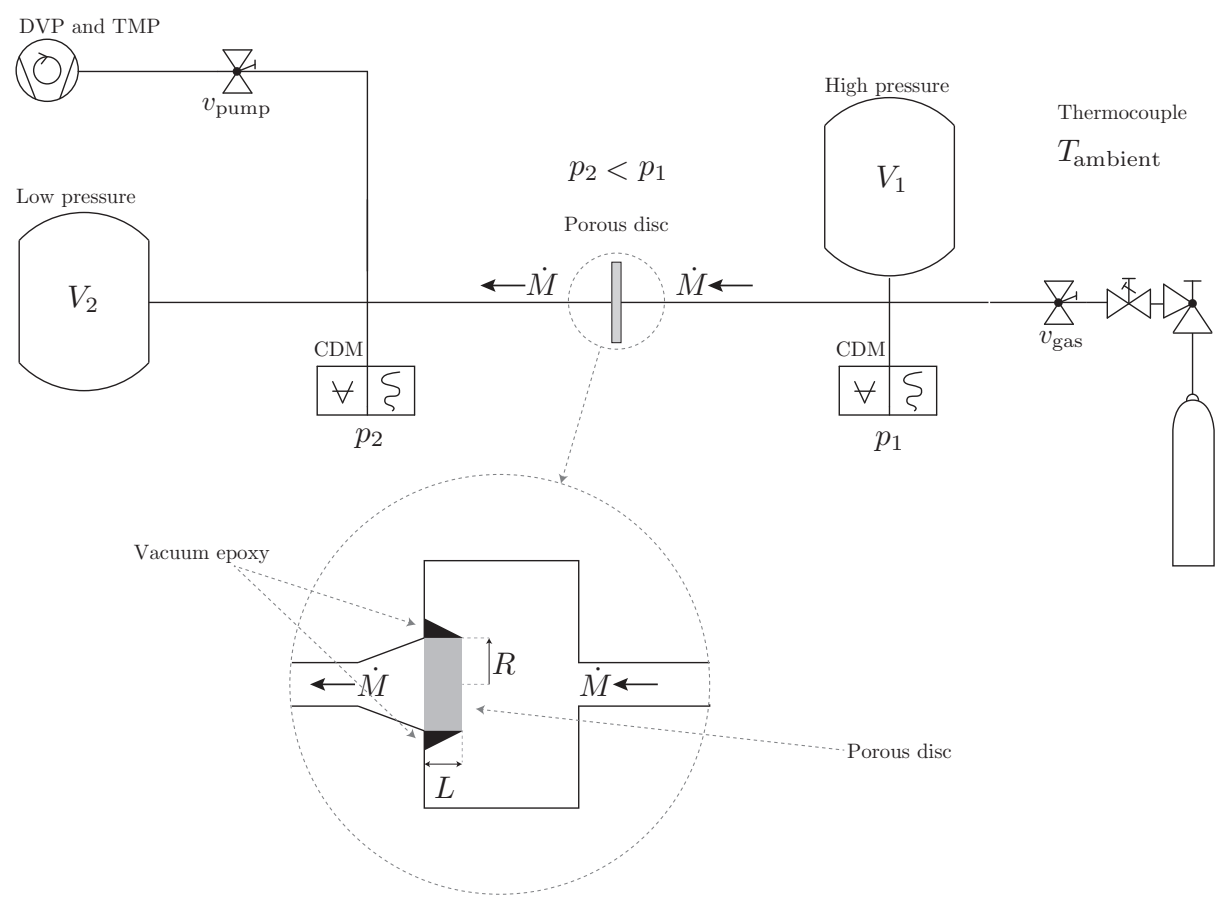

Figure 1: Schematic of the experimental setup. A diaphragm vacuum pump (DVP) and a turbomolecular pump (TMP) is connected to the experimental setup separated by a valve $v_{\text {pump. }}$. The high pressure and low-pressure side are separated by a porous ceramic media which is fixated with vacuum epoxy glue. Each side of the porous media has a reservoir with the highpressure tank volume of $V_{1}=255.8 \pm 5.5 \mathrm{~cm}^{3}$, low-pressure tank volume $V_{2}=238.8 \pm 5.1 \mathrm{~cm}^{3}$ and capacitance diaphragm manometers $(\mathrm{CDM})$ measuring pressure $p_{1}$ and $p_{2}$. The room temperature is measured with a thermocouple. The vacuum system is connected to a gas delivery system with four gas bottles, Helium, Neon, Nitrogen, and Argon. 
generated by setting an initial pressure drop between the reservoirs, see Fig. 1. This method requires very large tank volumes relative to the volume occupied by a gas inside a microporous medium: in our experimental setup this ratio is larger than $10^{3}$. The applied method is similar to the Brace method [1] (pulse decay method), usually used to analyze the permeability of the porous samples. In addition, in this work we take into consideration the effects of rarefaction. Even if the permeability can be deduced directly from the pressure (or pressure difference) variation in time, we prefer to start by providing the expressions of the mass flow rate through a microporous medium and the conditions of its derivation. The mass flow rate could be a useful quantity to characterize a porous sample; it can be used to derive the characteristic pore dimensions and the gas-surface interaction characteristics.

Under the quasi-steady conditions, i.e. when the flow through a porous medium is established, we assume that the gas temperatures in each tank, denoted $T_{1}$ and $T_{2}$, are in thermal equilibrium with the walls of the tanks and that both tanks are in thermal equilibrium with the environment. Therefore, we assume that the ambient temperature, denoted $T_{\text {ambient }}$, determines the gas temperatures $T_{1}$ and $T_{2}$ in each tank such that:

$$
T_{1} \approx T_{2} \approx T_{\text {ambient }}=T .
$$

A possible variation in temperature of the gas leaving and entering the tanks could directly perturb the significance of the measurement. To make this clearer, let us write the ideal gas law in each tank in the following form:

$$
p_{1} V_{1}=M_{1} \mathcal{R} T, \quad p_{2} V_{2}=M_{2} \mathcal{R} T,
$$

where $\mathcal{R}, p_{i}$, and $M_{i}, i=1,2$, are, respectively, the specific gas constant, the pressure and the mass of the gas in tank $i$. In the present study the maximum considered pressure is of the order of atmospheric pressure; therefore we do not consider here the real gas effects. However, the proposed approach can be generalized to take into account the real gas effects by using, for example, the van der Waals equation instead of the ideal gas law.

Under our experimental conditions, the volume of each reservoir is constant during an experiment, so it is possible to differentiate each expression in Eqs. (2), as it has been done in Ref [1]:

$$
\mathrm{d} M_{i}=\frac{V_{i}}{\mathcal{R} T} \mathrm{~d} p_{i}\left(1-\frac{\mathrm{d} T / T}{\mathrm{~d} p_{i} / p_{i}}\right), \quad i=1,2 .
$$

If the relative temperature variation in a tank is negligible in relation to the relative pressure variation in time, then the mass flow can be considered to be isothermal. Therefore, by defining a specific small time interval, $\mathrm{d} t$, it is then possible to obtain from Eqs. (3) the isothermal mass flow rates $\mathrm{d} M_{1} / \mathrm{d} t$ and $\mathrm{d} M_{2} / \mathrm{d} t$ as:

$$
\frac{\mathrm{d} M_{i}}{\mathrm{~d} t}=\frac{V_{i}}{\mathcal{R} T} \frac{\mathrm{d} p_{i}}{\mathrm{~d} t}, \quad \text { if } \quad \epsilon_{i}=\frac{\mathrm{d} T / T}{\mathrm{~d} p_{i} / p_{i}} \ll 1, \quad i=1,2 .
$$


Following the differentiation technique, we consider here the variation of any thermodynamic parameter, $\mathrm{d} M$ and $\mathrm{d} p$, sufficiently small to approximate $\mathrm{d} M / \mathrm{d} t$ and $\mathrm{d} p / \mathrm{d} t$ as the time derivative of the mass (i.e. mass flow rate $\dot{M}$ ) and the time derivative of the pressure, respectively. If the relative temperature variation is small compared to the pressure variation, the values of $\epsilon_{i}$ are small in Eqs. (4), so by adjusting the sign, $\mathrm{d} M_{i} / \mathrm{d} t$ can be considered as the mass flow rate $\dot{M}_{i}$ through the microporous media

$$
\dot{M}_{1}=-\frac{\mathrm{d} M_{1}}{\mathrm{~d} t}=-\frac{V_{1}}{\mathcal{R} T} \frac{\mathrm{d} p_{1}}{\mathrm{~d} t}, \quad \dot{M}_{2}=\frac{\mathrm{d} M_{2}}{\mathrm{~d} t}=\frac{V_{2}}{\mathcal{R} T} \frac{\mathrm{d} p_{2}}{\mathrm{~d} t} .
$$

In the frame of the quasi-stationary flow assumption, i.e. when the flow through a porous medium is established, it is clear that the mass flow rate leaving the first tank is necessarily equal to the mass flow rate entering the second tank and also to the mass flow rate at any point inside the porous medium

$$
\dot{M}_{1}=\dot{M}_{2}=\dot{M} .
$$

Along with this study, we will continue to admit relation (6) at any time. However, the assumption of the mass conservation, Eq. (6), neglects possible storage of gas in the porous medium. For the small ceramic porous sample used in present experiment, this hypothesis of the absence of the gas accumulation inside a porous medium is justified. In the same time, for the porous media such as the coal and shales, the compressible fluid storage needs to be accounted [5]. The experimental verification of mass conservation for the porous samples used in present experiments is discussed in Section 5.2.

Sometimes it is convenient to express the mass flow rate in function of the pressure difference between two tanks. From the mass conservation law, the mass of a gas leaving the first tank is necessarily equal to the mass of a gas entering the second tank, so Eq. (6) is valid. From Eqs. (5) and (6) we can obviously deduce:

$$
\dot{M}(t)=-\frac{V_{0}}{\mathcal{R} T} \frac{\mathrm{d}(\Delta p(t))}{\mathrm{d} t}, \quad V_{0}=\frac{V_{1} V_{2}}{V_{1}+V_{2}} .
$$

From the previous reasoning, it is clear that the mass flow rate can be calculated using expressions (5) and (7), when the pressure variation in each tank or the pressure difference between them in time is known. During the experiments, the pressure variations in time in each tank are measured and then fitted by using the exponential fitting function.

\subsection{Exponential pressure fitting}

The experimental procedure starts by setting the initial pressure difference $\Delta p_{0}$ between the tanks at time $t_{0}$ as:

$$
\Delta p_{0}=p_{1}\left(t_{0}\right)-p_{2}\left(t_{0}\right)=p_{01}-p_{02},
$$

where $p_{01}$ and $p_{02}$ are the initial pressures in the high and low-pressure tanks, respectively. This first step is done by opening the valve $v_{\text {pump }}$, see Fig. 1, 
for a short time, and then closing it. Further, we have a closed system with a pressure difference. Afterward, the gas begins to flow through a microporous medium from the high-pressure tank to the low-pressure tank up to the same final equilibrium pressure, $p_{\mathrm{f}}$, is reached in both tanks, see Fig. 2. The timedependent pressure difference between the tanks is noted as:

$$
\Delta p(t)=p_{1}(t)-p_{2}(t) .
$$

One example of the pressure evolution in each tank as well as of the pressure difference between two tanks is shown in Fig. 3.

Usually, when using the "pressure pulse technique", [1], the small pressure "pulses" (pressure differences between the tanks) are applied to the system, i.e. $\Delta p_{0} \ll p_{m}$, where $p_{m}=0.5\left(p_{1}+p_{2}\right)$ is the mean pressure between two tanks. We will discuss below that finally we do not really need to respect this restrictive condition and the an arbitrary pressure difference between the tanks can be used in the system if both tanks volumes are equal. However, the restriction for $p_{1} / p_{2}$ ratio exists when the tanks volumes are different, see Section 3.1.

The authors of Ref. [1] proposed to use a linear fit of the natural logarithm of an exponential function for the pressure variation in time, while we suggest to use directly the exponential pressure fit to describe the pressure difference decay in time in the following form:

$$
\Delta p(t)=\Delta p_{0} \exp \left(-\left(t-t_{0}\right) / \tau\right),
$$

where $\tau$ is the pressure relaxation time, which is constant during an experiment.

The same exponential representation of the pressure evolution in time in the first $p_{1}(t)$ and second $p_{2}(t)$ tanks is written in the form [1]:

$$
\begin{aligned}
& p_{1}(t)=p_{\mathrm{f}}+\left(p_{01}-p_{\mathrm{f}}\right) \exp \left(-\left(t-t_{0}\right) / \tau_{1}\right), \\
& p_{2}(t)=p_{\mathrm{f}}+\left(p_{02}-p_{\mathrm{f}}\right) \exp \left(-\left(t-t_{0}\right) / \tau_{2}\right),
\end{aligned}
$$

here $\tau_{1}, \tau_{2}$ are the gas pressure relaxation times in the reservoir 1 and 2 , respectively, $p_{\mathrm{f}}$ is the final pressure. The pressure variations with time $p_{i}(t)$ in each tank 1 and 2 can be thus associated with an exponential decay. In practice the pressure relaxation times $\tau_{i}$ are obtained from the fit of the measured pressure evolution in each tank, see Fig. 3. The properties of the pressure relaxation time are discussed in Section 4.

By using Eq. (7) we can now express the mass flow rate using the exponential representation of the pressure difference in time, Eq. (10), and then its analytical derivative, so the mass flow rate expression becomes

$$
\dot{M}(t)=-\frac{V_{0}}{\mathcal{R} T} \frac{\mathrm{d}(\Delta p(t))}{\mathrm{d} t}=\frac{V_{0}}{\mathcal{R} T} \frac{\Delta p_{0}}{\tau} \exp \left(-\frac{t-t_{0}}{\tau}\right) .
$$

From Eqs. (5) we can also express the mass flow rate using the exponential representations of the pressure variation in time in each tank, Eqs. (11), and 

here as well as the viscosity index $\omega$, are given in Table 1 .

Table 1: Parameters of the gases used in present experiments

\begin{tabular}{|c|c|c|c|c|}
\hline Gas & $\mu_{\text {ref }} \times 10^{-5}[$ Pa.s $]$ & $\omega$ & $\mathcal{R}[\mathrm{J} / \mathrm{kgK}]$ & Molar mass $\mathcal{M}\left[\mathrm{g} . \mathrm{mol}^{-1}\right]$ \\
\hline $\mathrm{He}$ & 1.865 & 0.66 & 2077.1 & 4.003 \\
$\mathrm{Ne}$ & 2.976 & 0.66 & 412.02 & 20.18 \\
$N_{2}$ & 1.656 & 0.74 & 296.80 & 28.00 \\
$\mathrm{Ar}$ & 2.117 & 0.81 & 208.13 & 39.95 \\
\hline
\end{tabular}

$$
\begin{gathered}
\dot{M}(t)=\dot{M}_{1}(t)=-\frac{V_{1}}{\mathcal{R} T} \frac{\mathrm{d}\left(p_{1}(t)\right)}{\mathrm{d} t}=\frac{V_{1}}{\mathcal{R} T} \frac{p_{01}-p_{\mathrm{f}}}{\tau_{1}} \exp \left(-\frac{\left(t-t_{0}\right)}{\tau_{1}}\right), \\
\dot{M}(t)=\dot{M}_{2}(t)=\frac{V_{2}}{\mathcal{R} T} \frac{\mathrm{d}\left(p_{2}(t)\right)}{\mathrm{d} t}=\frac{V_{2}}{\mathcal{R} T} \frac{p_{\mathrm{f}}-p_{02}}{\tau_{2}} \exp \left(-\frac{\left(t-t_{0}\right)}{\tau_{2}}\right) .
\end{gathered}
$$

Equations (12) and (13) provide the time-dependent expressions for the mass flow rate. Therefore, with one experiment we can calculate the mass flow rate for various different pressure ratios between the reservoirs, see Section 4 for more details. The conditions related to the implementation of this technique, as the choice of the tank volume size, possible pressure ratio and the impact of the thermal effects are discussed in Section 3.1.

\section{Relation with Darcy law and the permeability}

The Darcy law [1] allows us to relate the instantaneous discharge (or volumetric) flow rate through a porous medium, $Q$, to the pressure drop over a given distance $L$, which is the thickness of a porous sample:

$$
Q=\frac{K S}{\mu} \frac{\left(p_{1}-p_{2}\right)}{L},
$$

where $S$ is the surface of the porous sample, $\mu$ is the fluid viscosity, $K$ is the permeability. Initially, the Darcy law was derived for an incompressible fluid with constant viscosity and the permeability $K$ refers to the hydrodynamic (intrinsic permeability). In this article we use the Darcy law for the gases, i.e., a compressible fluid and we do not make any preliminary assumption of the rarefaction level of a gas.

The viscosity coefficient for a gas depends on gas temperature and gas nature and it is calculated as [1]:

$$
\mu=\mu_{\mathrm{ref}}\left(\frac{T}{T_{\mathrm{ref}}}\right)^{\omega},
$$

where $\omega$ is the gas viscosity index, $\mu_{\text {ref }}$ is the gas viscosity at temperature $T_{\text {ref }}=273.15 \mathrm{~K}$ [1]. The reference values of the viscosity, $\mu_{\text {ref }}$, for each gas used

For the liquid flows the volumetric flow rate is constant along a porous sample. For the gases only the mass flow rate is conserved along the porous

then their analytical derivatives, so the mass flow rate expressions become: 
sample. To express the permeability for a gas we can rewrite Eq. (14) in following form by replacing the pressure difference through a sample by the pressure gradient [5]:

$$
Q=-\frac{K S}{\mu} \frac{d p}{d x} .
$$

By using the relation between the volumetric and mass flow rate

$$
Q=\frac{\dot{M}}{\rho}=\dot{M} \frac{\mathcal{R} T}{p},
$$

then by integrating along the porous sample and by using the mass conservation property, we obtain the expression, analogous to Eq. (14), which relates the mass flow rate and the permeability

$$
\dot{M}=\frac{K S}{\mu} \frac{\Delta p}{L} \frac{p_{m}}{\mathcal{R} T} .
$$

Then, using Eqs. (5) and admitting the mass conservation again, Eq. (6), and using Darcy law in form (18), we can relate the pressure variation in each tank to the gas permeability:

$$
V_{1} \frac{\mathrm{d} p_{1}}{\mathrm{~d} t}=-p_{m} \frac{K}{\mu} \frac{S}{L}\left(p_{1}-p_{2}\right), \quad V_{2} \frac{\mathrm{d} p_{2}}{\mathrm{~d} t}=p_{m} \frac{K}{\mu} \frac{S}{L}\left(p_{1}-p_{2}\right) .
$$

To obtain previous relations we replaced the local pressure in Eq. (17) by the mean pressure, which does not vary during an experiment. This mean pressure is constant during an experimental run, when the tanks volumes are equal, $V_{1}=V_{2}$, and it varies only slightly when these volumes are slightly different, see Section 3.1, where the conditions of the mean pressure constancy are provided.

From Eqs. (19) we obtain the differential equation for the pressure difference $\Delta p(t)$ between the tanks:

$$
\frac{\mathrm{d}(\Delta p(t))}{\Delta p(t)}=-\frac{\mathrm{d} t}{\tau}
$$

where

$$
\tau=\frac{1}{p_{m}} \frac{\mu}{K} \frac{L V_{0}}{S} .
$$

This differential equation, subjected by the initial condition, $\Delta p\left(t=t_{0}\right)=\Delta p_{0}$, is easily solved and the variation in time of the pressure difference between the tanks is obtained in the form of Eq. (10).

From previous discussion it is clear that we do not use here any assumption about the smallness of the pressure "pulses" compared to the mean pressure in the tanks. Therefore, this technique can be implemented for any pressure difference between the tanks under the condition of equality of the tanks volumes. When the tanks volumes are different some restrictions have to be respected in order to keep the mean pressure close to a constant value during an experiment duration, see Section 3.1. In addition, to integrate the differential equation (20) the relaxation time $\tau$ has to be time independent. By analyzing Eq. (21) 
one can see that only mean pressure can be time-dependent. The experimental conditions of the mean pressure constancy in time are discussed in Section 3.1. The permeability depends on time only through the mean pressure, so the mean pressure constancy in time ensures that $\tau$ is constant in time and so justifies the integration of Eq. (20) and consequently the use of its solution, Eq. (10), for the experimental data (pressure) treatment. Therefore, the permeability $K$ of a porous sample can be derived directly from the pressure measurements.

The experimental curve of pressure variation in time can be fitted by exponential expression, Eq. (10), with $\tau$ as a fitting parameter. Then, the permeability can be found from the analytical expression for the pressure relaxation time $\tau$, Eq. (21), as:

$$
K=\frac{1}{p_{m}} \frac{\mu}{\tau} \frac{L V_{0}}{S} .
$$

Other expression for the permeability can be obtained from (18):

$$
K=\frac{\dot{M}}{p_{1}^{2}-p_{2}^{2}} \frac{2 \mu \mathcal{R} T L}{S}=\frac{\dot{M}}{\Delta p p_{m}} \frac{\mu \mathcal{R} T L}{S} .
$$

Both expressions, Eqs. (22) and (23), can be used to derive the permeability from the measurements.

\subsection{Conditions of constancy of mean pressure}

In this section we establish the conditions of the constancy of mean pressure during the measurement procedure, which leads to the pressure relaxation time constancy and justifies the use of expression (22) for the permeability calculations. In addition, as it has been pointed out in Ref. [8], in general case, the constancy of the mean pressure is an important point, especially for high pressure experiments, where the viscosity and compressibility factor may change as a function of pressure. In presented here experiments the implemented pressure and temperature conditions allow to us to stay under the ideal gas flow assumptions and the viscosity does not change with pressure. However, it is still important to have a constant mean pressure as for low permeable porous media the permeability can be a function of mean pressure due to rarefaction effects.

A pressure difference between the tanks is fixed initially, at $t_{0}$, equal to $\Delta p_{0}$, Eq. (8), then the gas flows through the porous medium up to the final stage, when a pressure equality in both tanks is reached, see Fig. 2. A relation between the pressure variation in each tank, i.e. from the initial pressure in each tank, $p_{i}\left(t_{0}\right), i=1,2$, to the final equilibrium pressure $p_{\mathrm{f}}$, reached in the system, can be calculated a priori as it is closely related to the tanks volumes ratio. From the ideal gas law and admitting again the mass conservation along the microporous medium at any time, we can write the following relation for two tanks if they are maintained at the same temperature

$$
\mathrm{d} p_{1} V_{1}=-\mathrm{d} p_{2} V_{2} .
$$


The previous relation is then integrated in time from an initial (at time $t_{0}$ ) state of a gas in each tank, $p_{i}\left(t_{0}\right)$, to its final state, $p_{\mathrm{f}}$. It is worth to underline that in Eq. (24) the expressions are exact (perfect) differential, and so their integration does not depend on the form (linear or exponential) of the pressure variation in time. Therefore, we obtain

$$
\frac{p_{\mathrm{f}}-p_{2}\left(t_{0}\right)}{p_{1}\left(t_{0}\right)-p_{\mathrm{f}}}=\frac{V_{1}}{V_{2}} .
$$

Without the loss of generality we can assume here that in the beginning of each experiment we have $p_{1}>p_{2}$. It is clear from Eq. (25) that by adjusting the tanks volumes ratio we could control the pressure variation between initial and final stages. From Eq. (25) we obtain the estimation of the maximal variation of the mean pressure $p_{\mathrm{m}}$ with time, from its initial value $p_{\mathrm{m}}\left(t_{0}\right)$ to its final value $p_{\mathrm{m}}\left(t_{\mathrm{f}}\right)=p_{\mathrm{f}}:$

$$
\frac{p_{\mathrm{f}}}{p_{\mathrm{m}}\left(t_{0}\right)}=\frac{p_{1}\left(t_{0}\right) V_{1}+p_{2}\left(t_{0}\right) V_{2}}{\left(V_{1}+V_{2}\right) p_{\mathrm{m}}\left(t_{0}\right)} .
$$

It is clear from Eq. (26) that when the tanks volumes are equal, the mean pressure $p_{\mathrm{m}}(t)$ does not vary in time, i.e., between its initial value, $p_{\mathrm{m}}\left(t_{0}\right)$, and its final value, $p_{\mathrm{f}}$, so $p_{\mathrm{m}}\left(t_{0}\right)=p_{\mathrm{f}}$. When the volumes are different we can estimate the maximal amplitude of mean pressure variation between its initial state $p_{\mathrm{m}}\left(t_{0}\right)$ and its final state $p_{\mathrm{f}}$, using Eq. (26) and the ratio of tanks volumes. To do this expression (26) can be rewritten as:

$$
\frac{p_{\mathrm{f}}}{p_{\mathrm{m}}\left(t_{0}\right)}=\frac{2\left(1+k_{V} k_{p}\right)}{\left(1+k_{V}\right)\left(1+k_{p}\right)} .
$$

We introduced here the tanks volumes ratio $k_{V}=V_{1} / V_{2}$ and the initial pressure ratio $k_{p}=p_{1}\left(t_{0}\right) / p_{2}\left(t_{0}\right)$. By using Eq. (27), we can calculate the variation of the mean pressure during an experiment, i.e. the ratio between its final and initial values $p_{\mathrm{f}} / p_{\mathrm{m}}\left(t_{0}\right)$.

In our experimental conditions two tanks volumes are related as $k_{V}=$ $V_{1} / V_{2}=1.071$, so from Eq. (27) we can find that for the initial pressure ratio between the tanks, $k_{p}$, equal to $1.5,2$ and 3 , the ratio $p_{\mathrm{f}} / p_{\mathrm{m}}\left(t_{0}\right)$ is equal 1.0069, 1.0114 and 1.0171, respectively. Therefore, the initial pressure ratio equal to 2 leads to approximately $1 \%$ of deviation of the mean pressure from its initial value. Under our experimental conditions the experimentally evaluated value of $p_{\mathrm{f}} / p_{\mathrm{m}}\left(t_{0}\right)$ was found lower than $1 \%$.

\section{Pressure relaxation time}

By fitting the measured time variation of the pressure difference between two tanks using exponential law, Eq. (10), we obtain a function $\Delta p(t)$ that describes the relaxation process with the help of a single fitting parameter $\tau$, that is the characteristic time of the experiment or pressure relaxation time. Similar expressions for the pressure variation in each tank, Eqs. (11), involve 
the relaxation parameters $\tau_{1}$ and $\tau_{2}$, which can also be obtained by the fitting of the pressure variation in one (high or low-pressure) tank. From different expressions of the mass flow rates, Eqs. (12) and (13), and using the mass conservation property in form (24), we can find that the ratios between the characteristic times are finally independent from the tanks volumes and are equal to one:

$$
\frac{\tau_{1}}{\tau_{2}}=\frac{\tau_{1}}{\tau}=\frac{\tau_{2}}{\tau}=1
$$

This analytical finding, Eq. (28), was confirmed experimentally. As it can be seen from Table 2 for the most cases the difference between three relaxation times, $\tau_{1}, \tau_{2}$ and $\tau$, is small, of the order of $1 \%$.

Finally, to obtain the mass flow rate through the microporous medium and its permeability, see Section 6, we can use either the exponential fit of the pressure difference between the tanks (in the case of the use of the differential pressure sensors), or just pressure evolution in a tank.

Table 2: Relaxation times $\tau_{1}, \tau_{2}$ and $\tau$, in seconds $(s)$ for the second disc, measured in the high and low-pressure tanks and by using the pressure difference between two tanks, respectively. For each of four gases the relaxation time is provided for two pressure differences between the tanks. The mean pressure of each experiment is given in fifth column. The last column provides the conduction time $\tau_{c}$, Eq.(29).

\begin{tabular}{|c|l|l|l|l|l|}
\hline & $\tau_{1}[\mathrm{~s}]$ & $\tau_{2}[\mathrm{~s}]$ & $\tau[\mathrm{s}]$ & $p_{\mathrm{m}}\left[10^{5} \mathrm{~Pa}\right]$ & $\tau_{\mathrm{c}}[\mathrm{s}]$ \\
\hline \hline \multirow{3}{*}{ HELIUM } & 54.11 & 53.94 & 54.03 & 1.13 & 0.96 \\
\cline { 2 - 6 } & 66.94 & 66.87 & 66.90 & 0.79 & 0.68 \\
\cline { 2 - 6 } & 153.83 & 155.60 & 154.72 & 0.04 & 0.03 \\
\hline \hline \multirow{3}{*}{ NEON } & 92.38 & 92.48 & 92.43 & 1.16 & 3.11 \\
\cline { 2 - 6 } & 119.98 & 118.59 & 119.28 & 0.79 & 2.13 \\
\cline { 2 - 6 } & 333.25 & 331.46 & 332.34 & 0.03 & 0.08 \\
\hline \hline \multirow{2}{*}{ NITROGEN } & 62.30 & 61.75 & 62.03 & 1.12 & 7.45 \\
\cline { 2 - 6 } & 92.98 & 92.04 & 92.50 & 0.70 & 4.64 \\
\cline { 2 - 6 } & 371.56 & 369.32 & 370.44 & 0.03 & 0.23 \\
\hline \hline ARGON & 79.27 & 78.61 & 78.94 & 1.11 & 8.11 \\
\cline { 2 - 6 } & 112.34 & 111.33 & 111.83 & 0.74 & 5.41 \\
\cline { 2 - 6 } & 444.07 & 444.22 & 444.15 & 0.04 & 0.26 \\
\hline
\end{tabular}

\subsection{Gas conduction time}

Now we can compare the gas conduction time $\tau_{c}$ to the gas relaxation time $\tau$ to have an additional estimation of the importance of the thermal effects. If the tank represents an infinite heat sink at constant temperature to the gas, then, the time it takes for the gas to reach equilibrium with the tank can be modeled. In Ref. [1] the transient heat conduction equation was solved analytically and the solution was presented as the infinite series of the Bessel functions. When keeping only the first leading term of the series the characteristic conduction time can be estimated as:

$$
\tau_{c}=\frac{\rho R_{r e s}^{2} P r}{2.4 \mu},
$$


where $\rho$ is the gas density, $R_{\text {res }}$ is the characteristic reservoir dimension, $\operatorname{Pr}$ is the Prandtl number. The reservoir characteristic dimension (its radius) is equal to $19.6 \mathrm{~mm}$, the Prandtl number is equal to $2 / 3$ and 0.71 , for the monoatomic and polyatomic gases, respectively. The gas conduction time, Eq. (29), is proportional to the gas density and so to the gas pressure under our experimental conditions. It depends also on the gas nature through the gas viscosity. The value of the gas conduction time for some experimental conditions are provided in Table 2, last column. For all considered cases the pressure relaxation time $\tau$ is much longer than the gas conduction time $\tau_{c}$. Therefore, we have a new experimental confirmation that the gas temperature remains close to the constant temperature during the measurements.

\subsection{Properties of the pressure relaxation time}

As the mass flow rate through a porous medium, Eqs. (12) and (13), and its permeability, Eq. (22), depend on the pressure relaxation time it is interesting to study its properties.

Figure 4 shows the pressure relaxation time, expressed in seconds, as a function of the inverse molecular mean free path, $\ell^{-1}$. The equivalent molecular mean free path is defined as following [2]:

$$
\ell=\frac{\mu v_{0}}{p_{\mathrm{m}}},
$$

where $v_{0}$ is the most probable molecular speed

$$
v_{0}=\sqrt{2 \mathcal{R} T} .
$$

It is clear from Eq. (30) that the inverse equivalent mean free path is a function of the mean pressure. By analyzing Fig. 4 and Table 2 we can conclude that the pressure relaxation time is proportional to the molar mass. That is, the shortest relaxation time is obtained for Helium, which has smaller molar mass, the longest relaxation time is found for Argon, which has greatest molar mass, see Table 1. All gases have similar behaviors as a function of the inverse molecular mean free path, which is a function of mean pressure. For the low mean pressure (large mean free path) the relaxation time is quasi-constant, then it decreases linearly with pressure increasing (the mean free path decreasing). This behavior is related to the number of collisions (molecule-molecule and molecule-wall collisions): when the intermolecular collisions are numerous (small mean free path) the relaxation time is short. With increasing of the molecular mean free path (decreasing of pressure) the number of intermolecular collision decreases which leads to the increase of the relaxation time, because the gas reaches its equilibrium state through the intermolecular collisions. When the molecular mean free path becomes large enough the number of the intermolecular collisions becomes negligible in comparison to the number of collisions with the wall (Knudsen diffusion regime) and the relaxation time becomes constant, see Fig. 4(b). In this case, the pressure relaxation time is determined only by the morphological 
parameters of a porous medium, i.e., mean pore size, porosity, tortuosity and particularities of gas-surface interaction.

It is worth to underline that the pressure relaxation time measured for two discs is different even if the same gas is considered. This fact lets us conclude that the internal structure of the microporous discs could be different. We comment on this observation in Section 6.3.

The pressure relaxation time can be normalized by the characteristic time of the flow, which is defined as follows:

$$
t_{c}=\frac{L}{v_{0}} .
$$

This characteristic time depends on the gas nature through the most probable gas velocity $v_{0}$, Eq. (31). The relaxation time, normalized by the characteristic time, is shown in Fig. 5, as previously in function of the inverse molecular mean free path. It is interesting to note that now all gases follow the same curve, so all gases have the same pressure relaxation time for the same value of the inverse molecular mean free path. Similar behavior of the relaxation time of the thermal creep flow was observed in Refs. [1], [1], [2], where the gas flow driven by only a temperature gradient through the microchannels of the circular and rectangular cross-sections was studied.

By taking into account the definition of the characteristic time, Eq. (32), we can rewrite expression of the permeability, Eq. (22), in the following form by using the equivalent molecular mean free path, Eq. (30),

$$
K=\frac{V_{0}}{S} \frac{t_{c}}{\tau} \ell .
$$

When the pressure relaxation time is used, the previous expression of the permeability allows calculating the permeability of a porous sample for different gases. This is because the normalized relaxation time is the same for all the considered here gases for a given value of molecular mean free path.

Another formula for the permeability can be derived by introducing gas relaxation time [2], $t_{f}$, which is inversely proportional to the collision frequency of the gas molecules, and it can be calculated as

$$
t_{f}=\frac{\mu}{p_{\mathrm{m}}}=\frac{\ell}{v_{0}},
$$

so the expression of permeability becomes

$$
K=\frac{V_{0} L}{S} \frac{t_{f}}{\tau} .
$$

Previous expression shows that the gas permeability depends on sample dimensions, $S$ and $L$, and on the tanks volume, $V_{0}$, used in experiments. The increasing (or decreasing) in this volume leads to the corresponding change in the pressure relaxation time, so that the ratio $V_{0} / \tau$ remains the same. Therefore, besides the geometrical characteristics, the permeability depends on the ratio between two characteristic times: gas and pressure relaxation times. 


\begin{tabular}{|l|l|l|l|l|l|}
\hline & $\frac{\delta V_{0}}{V_{0}}$ & $\frac{\delta \Delta p}{\Delta p}$ & $\frac{\delta T}{T}$ & $\varepsilon_{0}$ & $\frac{\delta \dot{M}}{\dot{M}}$ \\
\hline Uncertainty & $3.0 \%$ & $<1.9 \%$ & $<0.2 \%$ & $<1.2 \%$ & $3.6 \%-5.1 \%$ \\
\hline
\end{tabular}

Table 3: Measurement uncertainties of the mass flow rate, when the pressure difference between the tanks is used for the calculation. The maximal values obtained for two discs are given.

The mass flow rate was calculated from the pressure measurements in each tank by using the techniques, explained in Section 2.3. Three noble gases and one diatomic gas were used: Helium, Argon, Neon and Nitrogen.

\subsection{Measurement uncertainty of the mass flow rate}

The classical uncertainty calculation technique is used to estimate the measurement uncertainty of the mass flow rate, when it is calculated from the pressure or pressure difference exponential evolution, Eqs. (12) or (13). To ensure the upper limit of uncertainty, we use the maximum uncertainty on the mass flow rate measurements in the case of the pressure difference fit, Eq. (12), which reads:

$$
\frac{\dot{M}}{\dot{M}}=\frac{\delta V_{0}}{V_{0}}+\frac{\delta(\dot{\Delta p})}{\dot{\Delta p}}+\frac{\delta T}{T}+\varepsilon_{0}, \quad \text { where } \quad \frac{\delta(\dot{\Delta p})}{\dot{\Delta p}}=\frac{\delta \tau}{\tau}+\frac{\delta p_{1}}{p_{1}}+\frac{\delta p_{2}}{p_{2}} .
$$

In previous expression the uncertainty on the pressure difference in time involves the uncertainty on the fitting parameter $\tau$ and the uncertainty on the pressure sensors, provided by the manufacturer. The uncertainty on $\tau$ was obtained from the difference in magnitude of a 95\% confidence interval for $\tau$ to represent the experimental data. The parameter $\varepsilon_{0}$ in Eq. (36) represents the uncertainty coming from the non-isothermal effects [1] and it is equal to the maximum value in time of the ratio $(\mathrm{d} T / T) /(\mathrm{d} p / p)$. To evaluate the value of $\varepsilon_{0}$ the standard deviation and the mean temperature is used for $\mathrm{d} T / T$ term calculations, while the pressure difference and mean pressure are used to evaluate the pressure differential and pressure, respectively. The maximal relative uncertainties of each term in Eq. (36), obtained for two microporous discs, are summarized in Table 3. The uncertainty of the mass flow rate lies in the range $3.6-5.1 \%$.

\subsection{Results on the mass flow rate}

Typical pressure variations over time in both reservoirs are shown in Fig. 3. The exponential shape decay of the pressure in each tank and the pressure difference between two tanks are clearly visible on this figure. The exponential fit of the pressure (and pressure difference) variation curves during the total measurements duration with the pressure relaxation time as a single fitting parameter allow for very smooth reproduction of the experimental pressure recording.

\section{Mass flow rate}




\begin{tabular}{|l|l|l|l|l|l|l|l|}
\hline & $\frac{\delta V_{0}}{V_{0}}$ & $\frac{\delta L}{L}$ & $\frac{\delta S}{S}$ & $\frac{\delta \mu}{\mu}$ & $\frac{\delta p_{\mathrm{m}}}{p_{\mathrm{m}}}$ & $\frac{\delta \tau}{\tau}$ & $\frac{\delta K}{K}$ \\
\hline \hline Uncertainty & $3.0 \%$ & $0.5 \%$ & $0.1 \%$ & $1.0 \%$ & $<0.6 \%$ & $<1.4 \%$ & $5.0 \%-6.4 \%$ \\
\hline
\end{tabular}

Table 4: Measurement uncertainties of the microporous media permeability. The maximum of uncertainty of two discs is provided. microporous media lies in the range $5 \cdot 10^{-7}-5 \cdot 10^{-12}\left[\mathrm{~kg} \mathrm{~s}^{-1}\right]$. This range can be extended by modifying the experimental setup configuration.

The typical mass flow rate variations in time, calculated from the pressure variation in inlet tank, $-\dot{M}_{1}$, in outlet tank, $\dot{M}_{2}$, are shown in Fig. 6. It is clear that all three curves practically coincide, which confirms the mass conservation property within instrumental uncertainty.

\section{Permeability data}

\subsection{Measurement uncertainty on the permeability}

The uncertainty of the permeability measurements, when using Eq. (22), is calculated by the classical way similar to the calculation of the uncertainty on the mass flow rate, where the maximum uncertainty is used:

$$
\frac{\delta K}{K}=\frac{\delta V_{0}}{V_{0}}+\frac{\delta L}{L}+\frac{\delta S}{S}+\frac{\delta \mu}{\mu}+\frac{\delta p_{\mathrm{m}}}{p_{\mathrm{m}}}+\frac{\delta \tau}{\tau} .
$$

The relative measurement uncertainty on the permeability is presented in Table 4, where only the maximum values (of two porous discs) for each term of Eq. (37) are given, so the permeability uncertainty lies in the range $5.0-6.4 \%$.

\subsection{Permeability results}

The microporous media permeability, calculated using Eq. (22) from the measured mean pressure and pressure relaxation time, is shown in Fig. 7 as a function of inverse gas mean pressure. The results, obtained for two microporous discs and different gases, are presented and plotted in the log-log axis. Theoretically, for the large values of mean pressure, the permeability has to be constant, and the same for all gases tested for each disc. This part of the permeability curve corresponds to the classical Darcy law (intrinsic permeability), $K_{\infty}$, where a porous medium permeability does not depend on the nature of a fluid flowing inside. However, as it is clear from Fig. 7, only for the first disc with Helium this regime is reached in the present measurements, see "plateau" in Fig. 7(a). Larger mean pressure values, above atmospheric pressure, need to be applied to reach this regime for other gases and the second disc.

When the mean pressure decreases the permeability increases, and it becomes larger than the intrinsic permeability, the phenomenon of the apparent

In the current experimental setup the measured mass flow rate through the 
permeability appears, so-called Klinkenberg effect [2]. In this case, the permeability is usually expressed as

$$
K=K_{\infty}\left(1+\frac{b}{p_{m}}\right),
$$

where $b$ is a correction factor. As it is clear from Fig. 7 the apparent permeability becomes gas dependent: for a fixed mean pressure, the permeability is higher for lighter gases. However, if the same curves are plotted as a function of the mean free path, $\ell$, see Fig. 8, the data for different gases are located on the same curve. A similar property was also observed for the pressure relaxation time $\tau$, see Section 4.2 and Fig. 5 This finding leads to an interesting property: when the characteristic time is determined for a microporous medium for one gas, then the permeability can be calculated using Eq. (33) for other gases.

If we compare expression derived in the present paper to calculate the permeability, Eq. (22), with the classical expression of apparent permeability, Eq. (38), we find that Eq. (22) has the same asymptotic properties as Eq. (38), which can be confirmed by analyzing the measured data. When the mean pressure tends to infinity, then the product $\tau p_{m}$, tends to a constant value, see Fig. 9 and we find the constant intrinsic permeability. For the small values of the mean pressure $p_{m}$ the pressure relaxation time keeps its constant value, see Fig. 4, therefore the apparent permeability tends to infinity.

Recently several papers were published, where the dependence of the correction factor $b$ in Eq. (38) from the pressure is discussed [2], [2]. Usually the permeability is plotted as a function of inverse pressure in the linear-linear coordinate system. If we plot the experimental permeability curves, shown on Fig. 7, but using the linear-linear instead of logarithmic-logarithmic scale for both axis we can observe the typical behavior of the permeability: it seems to be increase linearly as the mean pressure decreases demonstrating the well-known Klinkenberg effect [2], [1]. From this evident linear dependence of the permeability on the mean pressure $\left(K=A+B / p_{m}\right)$ we could conclude that the correction factor $B$ is pressure independent and can be used for large pressure variation range. However, the linear-linear representation can mask some behavior, because it is difficult to present correctly the different order of magnitude of the parameter variation in linear scale. If we try to fit the whole permeability experimental curve with the same expression, $K=A+B / p_{m}$, we cannot find the same pair of $A$ and $B$ coefficients for the whole curve, see Fig. 11b). The relative deviation between the measured and fitted experimental points, $\left(K^{\exp }-K^{f i t}\right) / K^{f i t}$, for helium curve is shown of Fig. 12. It is clear that this curve fit very well the low pressure range and the large deviation exists in the high pressure (Darcy regime). This result demonstrates that the permeability points cannot be fitted with unique $b$ coefficient in the large pressure range when using the Klinkenberg expression. Of course, the more detailed study of this property is needed to find the numerical values of the correction factor $b$. 


\subsection{Difference between two discs}

Two porous discs considered in the present study were fabricated to have the same expected properties, i.e. the smallest average pore size of the order of $3 \mu$ $\mathrm{m}$. However, the measured permeability has very different values for two discs, especially for the low pressure. Therefore we assume that two discs have different internal structure. The tomographic analysis confirms this experimental finding: the averaged pore size was much larger for the first disc compared to the second one. Therefore, this technique can be used for the non-destructive analysis of the permeability of the microporous media. In addition this technique can be implemented to derive the characteristic pore size of a microporous sample.

\section{Conclusion}

The experimental procedure for the measurements of mass flow rate and permeability through the microporous media is proposed and analyzed. In the frame of this procedure the pressure evolution in each tank (or the evolution of the pressure difference between two tanks) is successfully fitted with an exponential function using one fitting parameter: the pressure relaxation time. The simple expressions for the mass flow rate and the permeability, derived from the exponential fitting of the pressure relaxation in each tank, are proposed. It was found that besides of the sample dimensions the gas permeability can be characterized by the ratio between gas relaxation time (inverse of gas collision frequency) and the pressure relaxation time. With the present experimental setup we measured the mass flow rate in the range $5 \cdot 10^{-7}-5 \cdot 10^{-12}[\mathrm{~kg} / \mathrm{s}]$ and the permeability in the range $10^{-14}-10^{-11}\left[\mathrm{~m}^{2}\right]$. However, we are not restricted to these ranges of parameters with the present experimental setup (volume configuration and sample size). We estimate that we could measure at least 50 times lower mass flow rate and permeability. To go further in low permeability measurements the experimental setup has to be modified. The proposed approach is the first very promising stage to evolve towards measurements of even lower permeabilities and also the characteristic dimension (pore size) of membranes used for microfiltration $(>100 \mathrm{~nm})$ and ultrafiltration $(>10$ $\mathrm{nm})$.

\section{Acknowledgment}

This work has been carried out in the framework of the Labex MEC (ANR10-LABX-0092) and of the A*MIDEX project (ANR-11-IDEX-0001-02), funded by the "Investissements d'Avenir" French Government program managed by the French National Research Agency (ANR). The authors (M.V. Johansson, P. Perrier, and I. Graur) would like to acknowledge financial support provided by the European Union network program H2020, MIGRATE project under Grant Agreement No.643095. 
[1] S. P. Adiga, C. Jin, L. A. Curtis, N. A. Monteiro-Riviere, and R. J. Narayan, "Nanoporouse membranes for medical and biological applications," WIREs Nanomedicine and Nanobiotechnology Advances Reviews, vol. 1, no. 568$581,2009$.

[2] R. Abedini and A. Nezhadmoghadam, "Application of membrane in gas separation processes: its suitability and mechanisms," Petroleum and Coal, vol. 52 , p. $69,2010$.

[3] Y. S. Lin and A. J. Burggraaf, "Experimental studies on pore size change of porous ceramic membranes after modification," Journal of Membrane Science, vol. 79, pp. 65-82, 1993.

[4] M. E. Naraghi and F. Javadpour, "A stechastic permeability model for the shal-gas system," International Journal of Goal Geology, vol. 14, pp. 111$124,2015$.

[5] R. Sander, Z. Pan, and L. D. Connell, "Laboratory measuremant of low permeability unconventional gas reservoir rocks: A review of experimental methods," Journal of Natural Gas Science and Engineering, vol. 37, pp. 248-279, 2017.

[6] Y. Jannot and D. Lasseux, "A new quasi-steady method to measure gas permeability of weakly permeable perouse media," Review of scientific instruments, vol. 83, p. 015113, 2012.

[7] G. H. Bruce, D. Peaceman, H. Rachford Jr, J. Rice, et al., "Calculations of unsteady-state gas flow through porous media," Journal of Petroleum Technology, vol. 5, no. 03, pp. 79-92, 1953.

[8] W. F. Brace and R. J. Martin, "A test of the low effective stress for cristalline rocks of low porosity," Int. J. Rock. Mech. Min. Sci, vol. 5, no. 415-426, 1968.

[9] E. Dana and F. Skoczylas, "Gas relative permeability and pore structure of sanstones," International Journal of Rock Mechanics and Mining Sciences, vol. 36, pp. 613-625, 1999.

[10] J. Billiotte, D. Yang, and K. Su, "Experimental study on gas permeability of mudstones," Physics and Chemistry of the Earth, vol. 33, pp. 5231-5236, 2008.

[11] M. Rojas Cardenas, I. Graur, P. Perrier, and J. G. Méolans, "Thermal transpiration flow: a circular cross-section microtube submitted to a temperature gradient," Phys. Fluids, vol. 23, p. 031702, 2011.

[12] M. Rojas-Cardenas, I. Graur, P. Perrier, and J. G. Méolans, "An experimental and numerical study of the final zero-flow thermal transpiration stage," J Therm. Sci. Technol., vol. 7, pp. 437-452, 2012. 
[13] M. Rojas-Cardenas, I. Graur, P. Perrier, and J. G. Méolans, "Timedependent experimental analysis of a thermal transpiration rarefied gas flow," Phys. Fluids, vol. 25, p. 072001, 2013.

[14] T. Ewart, P. Perrier, I. A. Graur, and J. G. Méolans, "Mass flow rate measurements in gas micro flows," Experiments in Fluids, vol. 41, no. 3, pp. 487-498, 2006.

[15] T. Ewart, P. Perrier, I. A. Graur, and J. G. Méolans, "Mass flow rate measurements in microchannel, from hydrodynamic to near free molecular regimes," Fluid mechanics, vol. 584, pp. 337-356, 2007.

[16] W. F. Brace, J. B. Walsh, and W. T. Frangos, "Permeability of granite under high pressure," Journal of Geophysical Research, vol. 73, no. 6, pp. $2225-2236,1968$.

[17] D. D. Do, Adsobtion analysis: equilibria and kinetics, vol. 2. Imperial College London SW7 2BT: Imperial College Press, 1998.

[18] G. A. Bird, Molecular Gas Dynamics and the Direct Simulation of Gas Flows. Oxford Science Publications, Oxford University Press Inc., New York, 1994.

[19] E. B. Arkilic, M. A. Schmidt, and K. S. Breuer, "Gaseous slip flow in long microchannels," J. Microelectromech. S., vol. 6, no. 2, pp. 167-178, 1997.

[20] F. Sharipov, Rarefied gas dynamics. Fundamentals for research and practice. WILEY-VCH Verlag GmBH \& Co. KGaA. Weinheim, 2015.

[21] H. Yamaguchi, M. Rojas-Cardenas, P. Perrier, I. Graur, and T. Niimi, "Thermal transpiration flow through a single rectangular channel," Journal of Fluid Mechanics, vol. 744, pp. 169-182, 2014.

[22] C. Cercignani, Mathematical methods in kinetic theory. Premuim Press, New York, London, 1990.

[23] L. J. Klinkenberg, "The permeability of porous media to liquid and gases," Drilling and Production Practice, Amarican Petroleum Institute, pp. 200213, 1941.

[24] D. Lasseux, F. J. Valdes Parade, and M. Porter, "An improved macroscale model for gas slip dlow in porous media," Journal of Fluid Mechanics, vol. 805, pp. 118-146, 2016.

[25] L. Wu, M. T. Ho, L. Germanou, X.-J. Gu, C. Liu, K. Xu, and Y. Zhang, "On the apparent permeability of porous media in rarefied gas flows," Journal of Fluid Mechanics, vol. 822, pp. 398-417, 2016. 


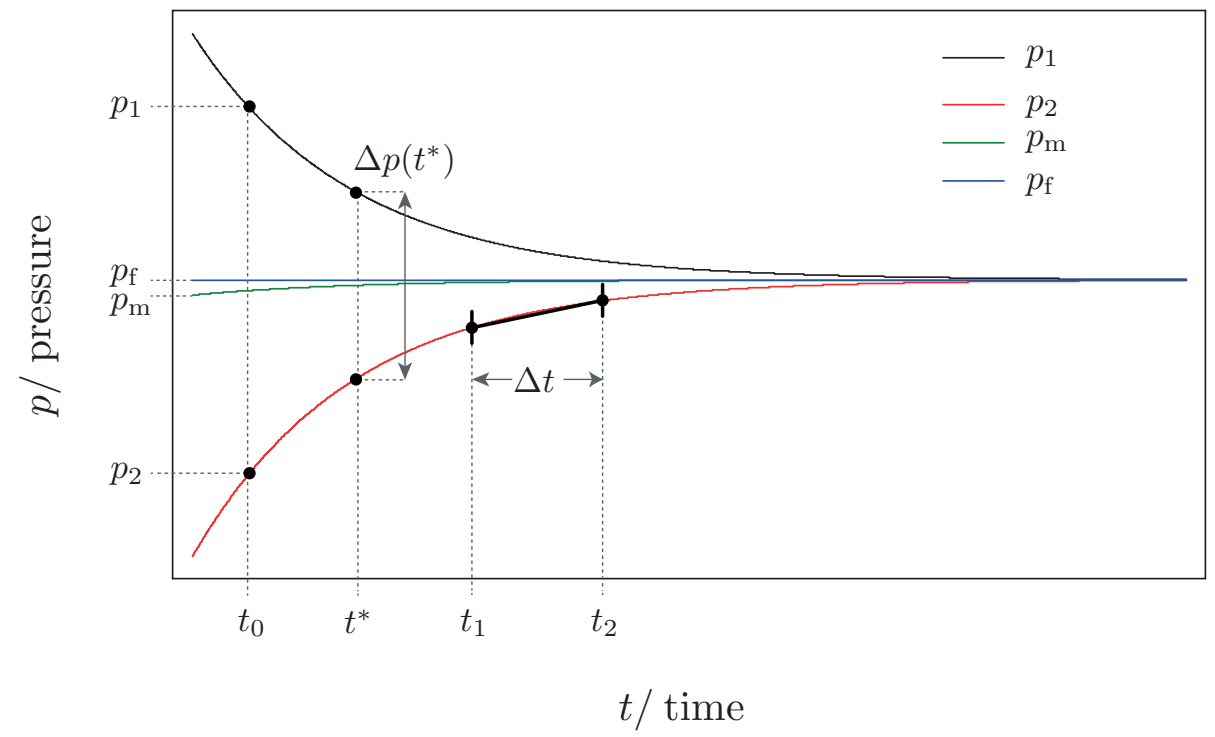

Figure 2: Schematic representation of upstream, $p_{1}$, and downstream, $p_{2}$, pressure response as a function of time. Where $p_{\mathrm{m}}$ denotes mean pressure and $p_{\mathrm{f}}$ the final mean pressure. 


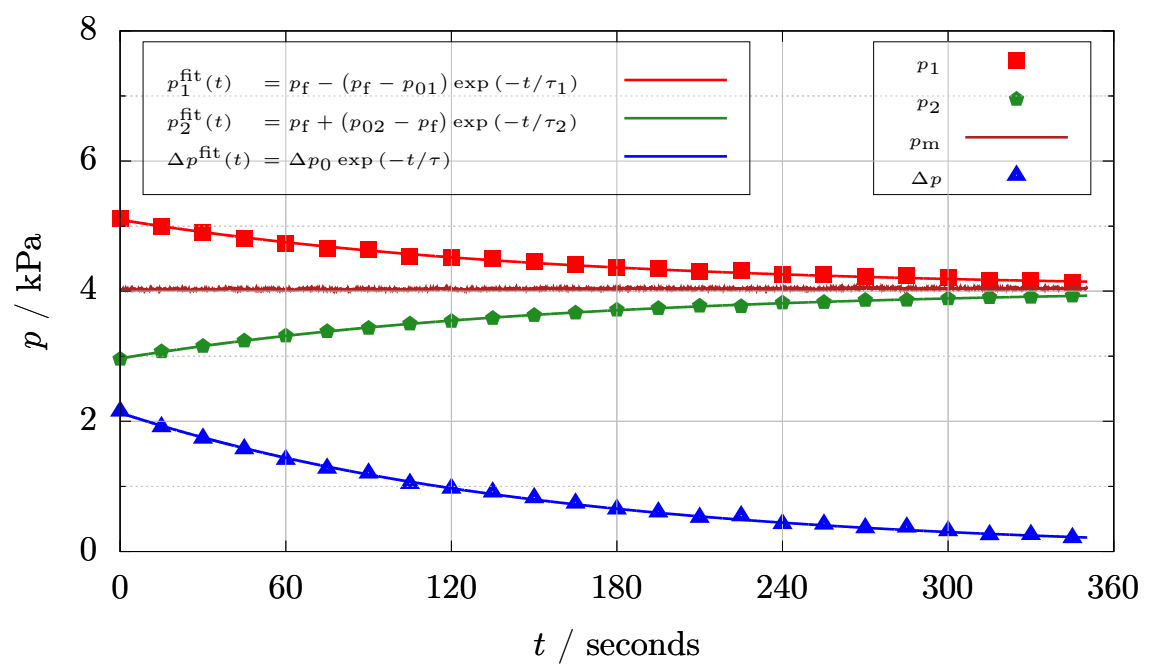

Figure 3: Color on-line: pressure evolution as a function of time. Red squares $\square$ is upstream tank pressure, $p_{1}$, green pentagons $\square$ is the downstream tank pressure, $p_{2}$, magenta line - is the mean pressure, blue triangles $\triangle$ is the pressure difference between tanks. Red, green and blue lines represent the exponential pressure fits, which correspond to Eqs. (11) and (10). 


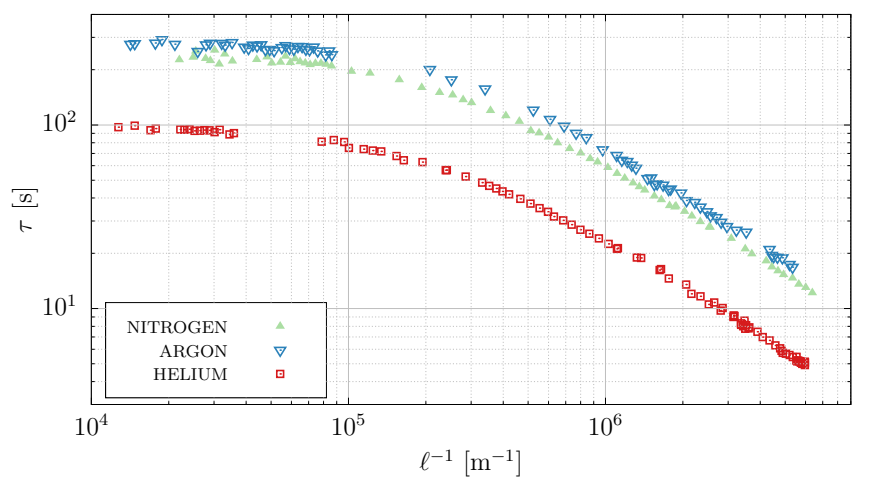

(a)

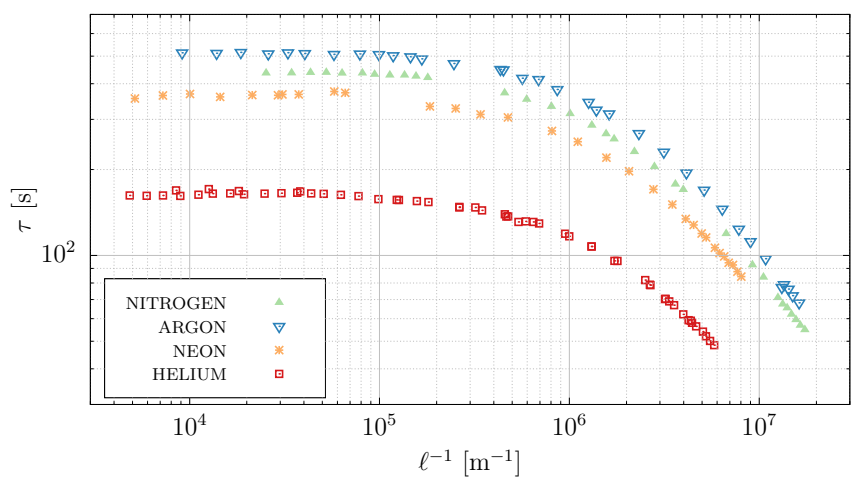

(b)

Figure 4: Color on-line: variation of the relaxation time as a function of inverse molecular mean free path, $\ell^{-1}$, for different gases and two porous media: (a) first disc, (b) second disc. 


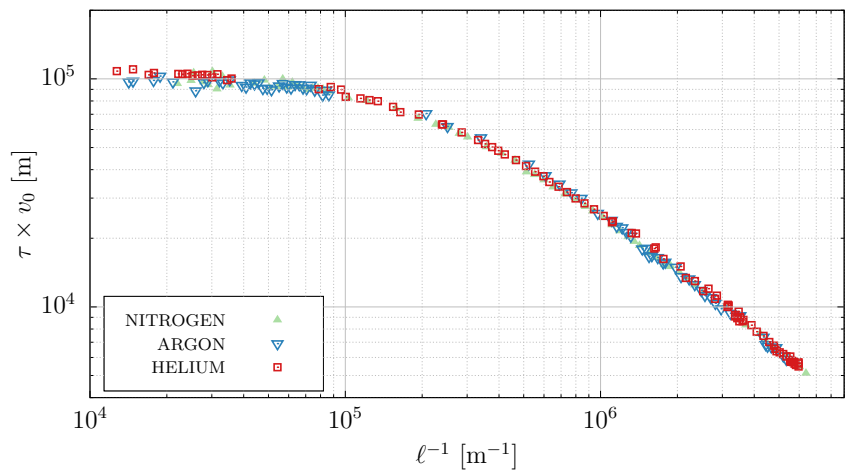

(a)

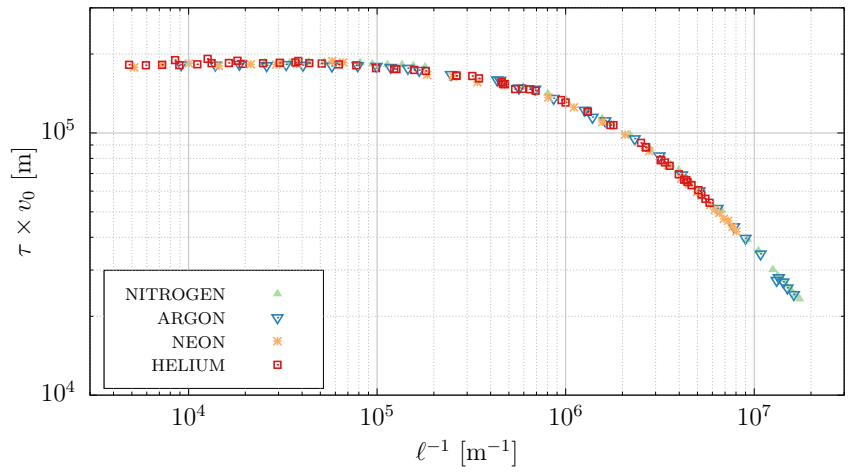

(b)

Figure 5: Color on-line: variation of the relaxation time, normalized by the characteristic time, Eq. (32), as a function of inverse molecular mean free path, $\ell^{-1}$, for different gases and two porous media: (a) first disc, (b) second disc. 


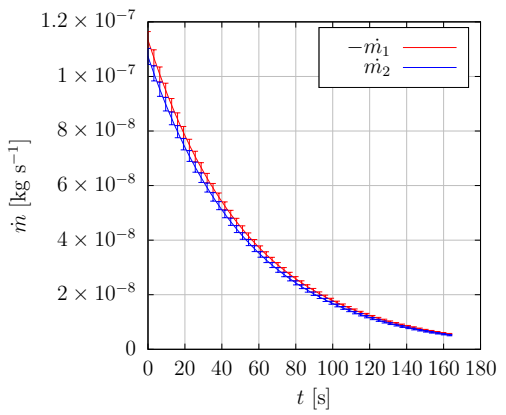

(a)

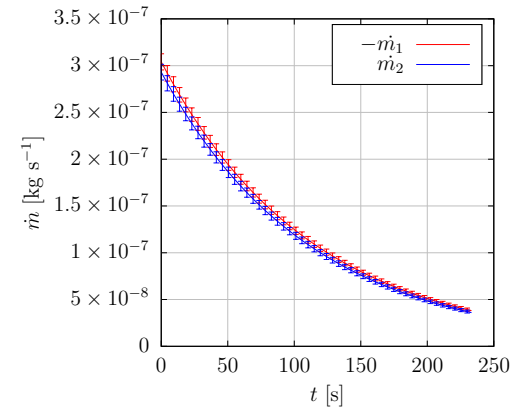

(b)

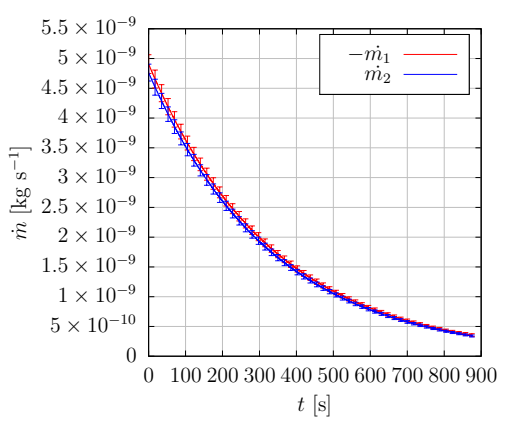

(c)

Figure 6: Color on-line: three examples of the mass flow rate variation, calculated from the pressure variation in the first tank, $\dot{M}_{1}$, in the second tank, $\dot{M}_{2}$. a) Helium at $p_{\mathrm{m}}=1.13 \times 10^{5}$ $\mathrm{Pa}$, first row in Table 2, b) Argon at $p_{\mathrm{m}}=0.74 \times 10^{5} \mathrm{~Pa}$, row 11 in Table $2 \mathrm{c}$ ) Neon at $p_{\mathrm{m}}=0.03 \times 10^{5} \mathrm{~Pa}$, row 6 in Table 2 . 


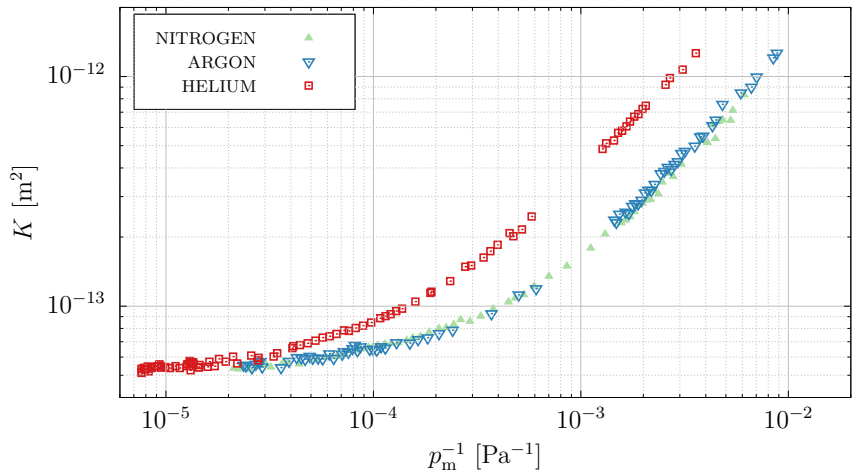

(a)

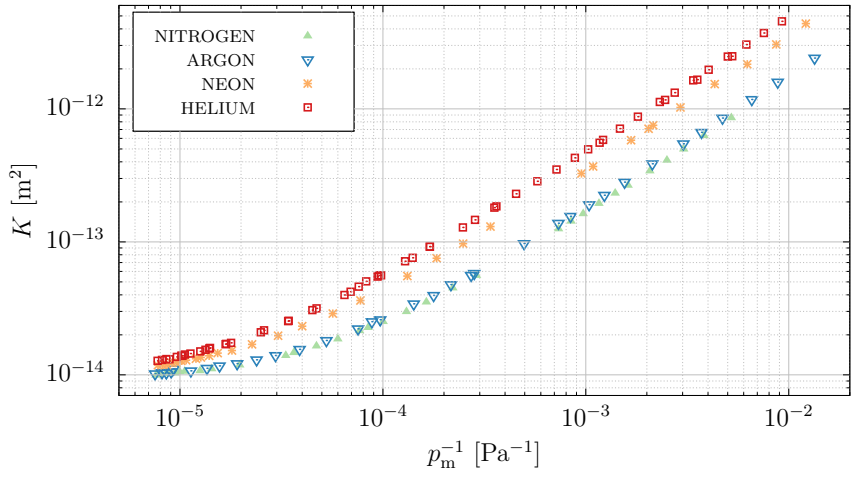

(b)

Figure 7: Color on-line: variation of the permeability of micro porous media, as a function of inverse mean pressure for four gases and two porous media: (a) first disc, (b) second disc. 


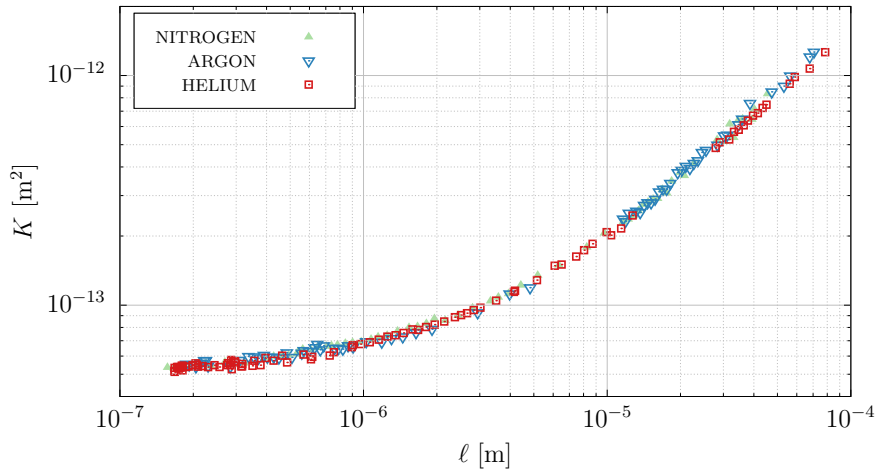

(a)

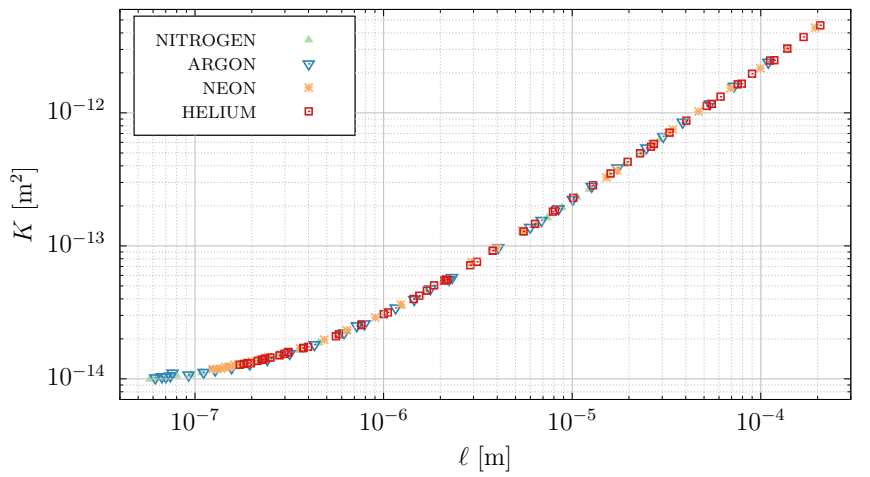

(b)

Figure 8: Color on-line: variation of the permeability of micro porous media, as a function of mean free path for four gases and two porous media: (a) first disc, (b) second disc. 


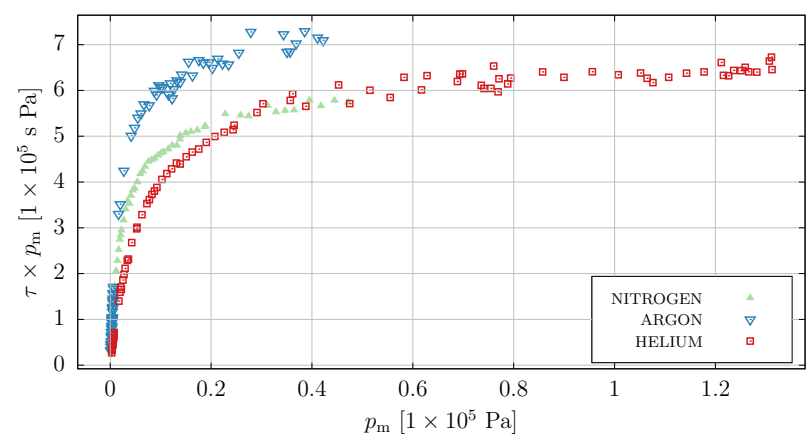

(a)

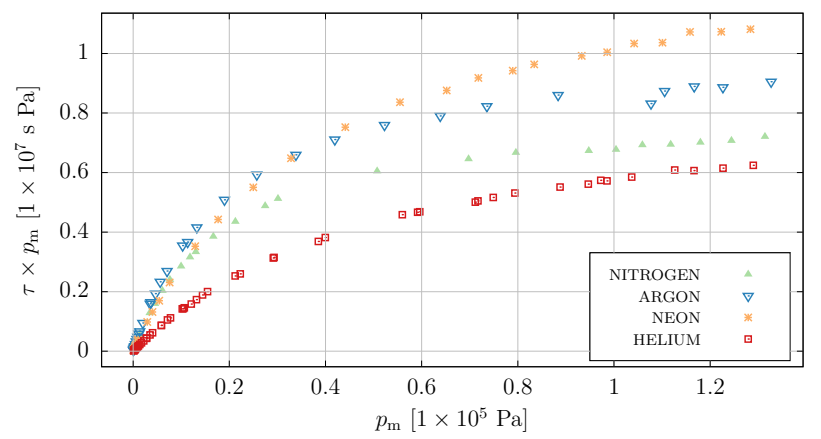

(b)

Figure 9: Color on-line: variation of the quantity $\tau p_{m}$ as a function of mean pressure for two porous media: (a) first disc, (b) second disc. 


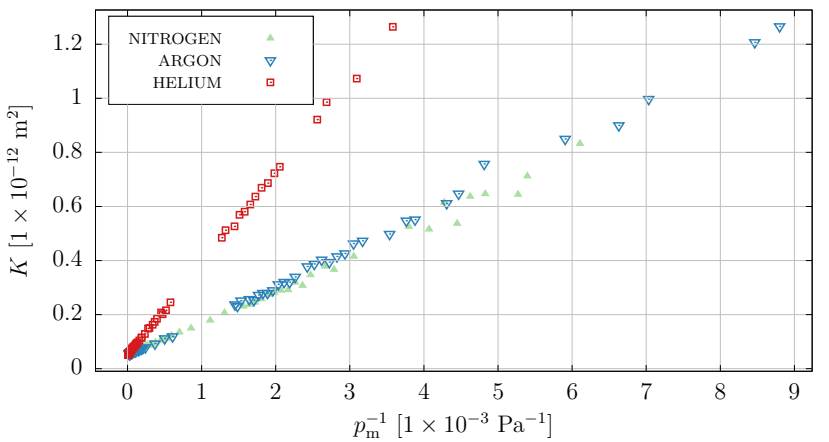

(a)

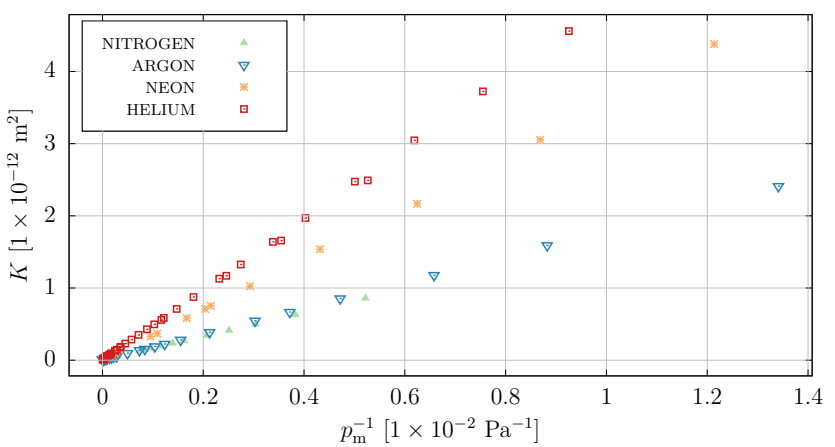

(b)

Figure 10: Color on-line: Permeability of micro porous media, Eq. (22), as a function of inverse mean pressure for four gases and two porous media: (a) first disc, (b) second disc. 


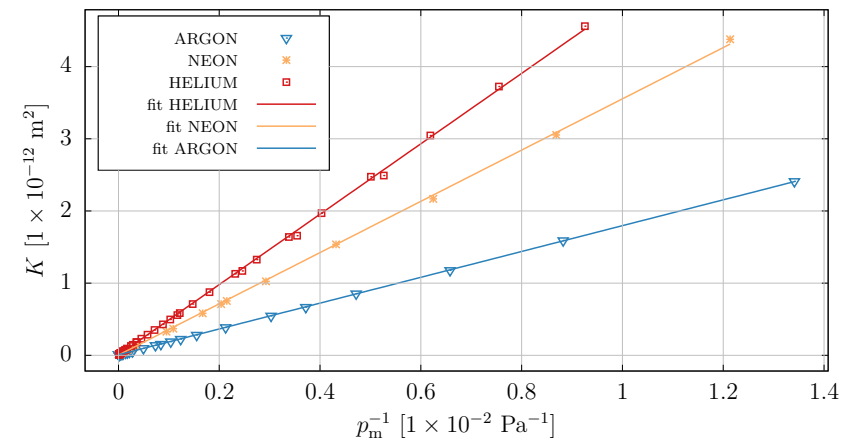

(a)

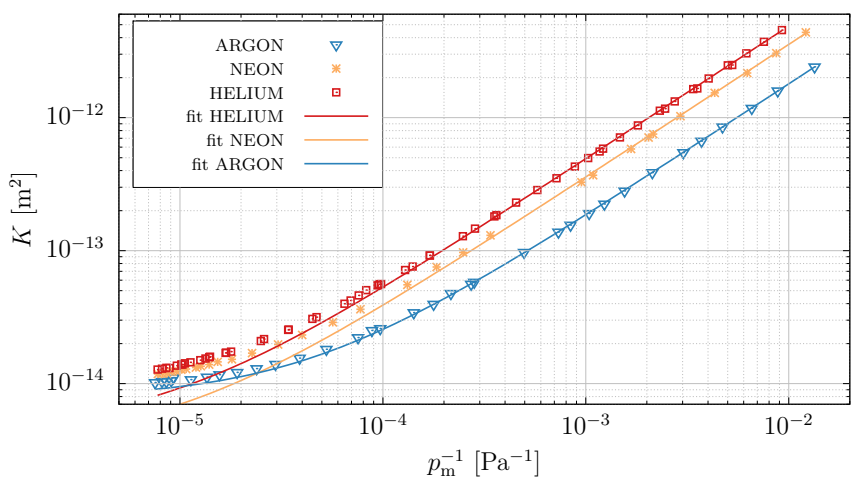

(b)

Figure 11: Color on-line: (a) measured permeability for three gases (symbols) for the first disc with the linear fit of the experimental data (solid lines); (b) the same data as on (a) but plotted in log-log coordinates.

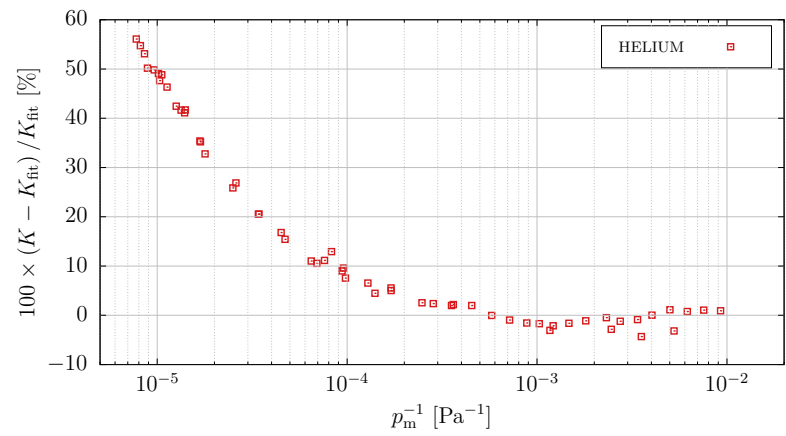

(a)

Figure 12: Color on-line: the relative error for the permeability: $\left(K_{\exp }-K_{f i t}\right) / K_{f i t} \times 100 \%$ for the measurements of Helium for the first disc, $K_{\exp }$ is the measured value, $K_{f i t}$ is the fitted value. The same experimental points and linear fit are also presented on Fig. 11. 